\title{
Cloning and Sequencing Analysis of the Repressor Gene of Temperate Mycobacteriophage L1
}

\author{
Subrata Sau*, Partho Chattoraj, Tridib Ganguly, Chia Yen Lee ${ }^{\dagger}$ and Nitai Chandra Mandal* \\ Department of Biochemistry, Bose Institute, P1/12-CIT Scheme VII M, Calcutta 700 054, India \\ 'Department of Microbiology, Molecular Genetics and Immunology, KU Medical Center, \\ 3901 Rainbow Blvd., Kansas City, KS 66103, USA
}

Received 24 June 2003, Accepted 13 August 2003

\begin{abstract}
The wild-type and temperature-sensitive (ts) repressor genes were cloned from the temperate mycobacteriophage L1 and its mutant L1cIts391, respectively. A sequencing analysis revealed that the $131^{\text {st }}$ proline residue of the wildtype repressor was changed to leucine in the ts mutant repressor. The $100 \%$ identity that was discovered between the two DNA regions of phages $\mathrm{L1}$ and L5, carrying the same sets of genes including their repressor genes, strengthened the speculation that $\mathrm{L} 1$ is a minor variant of phage $L 5$ or vice versa. A comparative analysis of the repressor proteins of different mycobacteriophages suggests that the mycobacteriophage-specific repressor proteins constitute a new family of repressors, which were possibly evolved from a common ancestor. Alignment of the mycobacteriophage-specific repressor proteins showed at least 7 blocks (designated I-VII) that carried 3-8 identical amino acid residues. The amino acid residues of blocks V, VI, and some residues downstream to block VI are crucial for the function of the $\mathrm{L} 1$ (or L5) repressor. Blocks I and II possibly form the turn and helix 2 regions of the HTH motif of the repressor. Block IV in the L1 repressor is part of the most charged region encompassing amino acid residues 72-92, which flanks the putative $\mathrm{N}$ terminal basic (residues 1-71) and C-terminal acidic (residues 93-183) domains of $\mathrm{L} 1$ repressor.
\end{abstract}

Keywords: Helix-turn-helix (HTH) motif, M. smegmatis, Mycobacteriophage L1, Repressor gene, Temperaturesensitive (ts)

*To whom correspondence should be addressed.

Fax: 91-33-2337-9416

E-mail: sau@bic.boseinst.ernet.in or ncmandal@boseinst.ernet.in

\section{Introduction}

The phages of mycobacteria are extremely diverse in nature and carry highly mosaic genomes (Pedulla et al., 2003). Several molecular tools have been developed from mycobacteriophages during the last ten years. They are very useful for mycobacterial research and the diagnosis of mycobacterial infections (Hatfull, 2000). Among the mycobacteriophages, L5, Bxb1, I3 (Hatfull, 2000), Ms6 (Garcia et al., 2002), and L1 (Chaudhuri et al., 1993) were studied to some extent at the molecular level. Both the cis- and trans-acting regulatory elements that are involved in the integration of L5 into its host genome were identified and characterized at length (Hatfull, 2000). Several promoters of I3 (Ramesh and Gopinathan, 1995), L5 (Nesbit et al., 1995), Bxb1 (Jain and Hatfull, 2000), and Ms6 (Garcia et al., 2002) were reported. The repressors of both L5 and Bxb1 negatively regulate the expression of their respective early promoters by binding at the cognate operators (Nesbit et al., 1995; Jain and Hatfull, 2000). Currently, the molecular mechanism of the interaction between the repressor of any mycobacteriophage and its operator DNA is poorly understood, though it has immense potential in deciphering the gene regulation in both mycobacteriophage and mycobacterial systems. Also, the information could lead to the construction of the tightly regulated expression vector (for the mycobacterial system) by assembling the early promoter and the repressor gene of temperate mycobacteriophage.

Mycobacteriophage L1, a sister homoimmune phage of L5, has a 50-kb double-stranded DNA genome. The genes that regulate both the lysogenic and lytic development of L1 were mapped and some were to some extent characterized (Chaudhuri et al., 1993). The G27 gene of L1was shown to be an early positive regulator as it controls the expression of both the delayed early and late genes at the transcriptional level (Datta and Mandal, 1998). A few promoters of L1 have been cloned in a promoter-cloning vector having $\beta$-galactosidase as 
the reporter gene, but further characterization of these promoters is yet to be reported (Barletta et al., 1992). Recently, two early promoters of L1 were cloned, which are regulated by its own repressor (Chattopadhyay et al., 2003). In this communication, as a prerequisite to developing suitable mycobacteria-specific expression vectors and studying the molecular nature of the DNA-protein interaction in the mycobacterial system, we report the cloning and preliminary characterization of the repressor genes of both wild-type L1 and its $c \mathrm{I}$ ts391 mutant derivative (Chaudhuri et al., 1993).

\section{Materials and Methods}

Bacterial and phage strains, vectors and growth conditions $E$. coli $\mathrm{DH} 5 \alpha$ cells were grown in Luria broth. M. smegmatis mc $^{2} 155$ and LR222, obtained from Dr. Anil Tyagi (University of Delhi, South Campus, New Delhi, India), were routinely grown in Middlebrook 7H9 medium (Chaudhuri et al.,1993). The wild-type mycobacteriophage L1, its mutants $\mathrm{L} 1 c \mathrm{I}^{-}$, L1cIts391, and its lysogen were described in Chaudhuri et al. (1993). The E. coli-M. smegmatis shuttle vector pSD5S30, containing a mycobacteriaspecific promoter S30, was obtained from Dr. Anil Tyagi (Bashyam et al., 1996; Jain et al., 1997).

DNA isolations and manipulations All of the molecular biological and recombinant DNA techniques were used as described by Sambrook et al. (1989). Mycobacteriophage L1 DNA was isolated as described by Chaudhuri et al. (1993). Isolation of plasmid DNA from M. smegmatis and the electrotransformation of M. smegmatis LR222 were performed according to the standard methods of Das Gupta et al. (1993).

Cloning of the wild-type repressor gene of mycobacteriophage L1 L1 DNA was digested with PstI and ligated to the identical site of pSD5S30. The ligated DNA was electroporated into competent M. smegmatis LR222 (L1cIts391), then the culture was plated on Luria agar containing kanamycin $(25 \mu \mathrm{g} / \mathrm{ml})$. After incubation for $20 \mathrm{~h}$ at $32^{\circ} \mathrm{C}$, when the transformants were just visible, the plates were shifted to $42^{\circ} \mathrm{C}$ and further incubated for 20$24 \mathrm{~h}$. The colonies, which grew larger at $42^{\circ} \mathrm{C}$, were selected and purified. The ones that showed immunity to superinfection by $\mathrm{Licl}^{-}$ were selected and purified. Plasmids were isolated from three of these transformants and digested with PstI. The fragments were analyzed by $1 \%$ agarose gel electrophoresis.

Construction of a limited genomic library of L1cIts391 The genomic DNA of L1cIts391 was digested together with BamHI \& SalI. The DNA fragments with a size range from 1,000 to $2,000 \mathrm{bp}$ were eluted from low melting gel. Fragments were ligated into identical sites of pBluescript SK. DNAs were then transformed to E. coli XL1 Blue. Several transformants, which appeared as colorless colonies on LA-Amp-X-gal-IPTG, were selected and purified. Plasmids, isolated from twenty such colorless colonies, were digested by BamHI \& SalI and analyzed by agarose gel electrophoresis (data not shown). Those carrying the $1.4 \mathrm{~kb}$ Bam HISalI fragments were selected and purified.
DNA sequencing and analysis DNA sequencing of the L1 inserts were carried out by $\mathrm{ABI}$ automated sequencer according to the manufacturer's protocol.

DNA accession number Upon deposition of the $1.4 \mathrm{~kb}$ L1 DNA sequence (of pCP2) carrying the ts mutant repressor gene of phage L1, the accession number AY303696 was obtained from GenBank.

\section{Results and Discussion}

Cloning of the DNA fragment carrying the wild-type repressor gene of phage $\mathrm{L} 1$ In temperate mycobacteriophage L1, the gene encoding repressor has been designated $c$ I (Chaudhuri et al., 1993). The cIts391 mutation, which makes the repressor phenotypically temperature sensitive, causes the induction of L1cIts391 lysogen in M. smegmatis at $42^{\circ} \mathrm{C}$, thereby killing the bacterium (Chaudhuri et al., 1993). It was assumed that the wild-type repressor of L1 that was supplied in trans from a plasmid in a lysogen of L1cIts391 could prevent the induction (killing) of the latter at $42^{\circ} \mathrm{C}$. Therefore, for cloning the repressor gene of phage L1, a PstI genomic library of L1 DNA was prepared by ligating PstI-digested L1cI DNA fragments with the same enzyme-digested plasmid DNA and transformed into M. smegmatis LR222 lysogenic for L1cIts391. The resulting transformants, which survived and grew on a kanamycin plate at $42^{\circ} \mathrm{C}$, were selected and purified. A restriction analysis of the plasmids from three of these transformants showed that all of them carried a common $6 \mathrm{~kb}$ PstI fragment of L1 DNA (data not shown). This plasmid was designated pLC1 (Table 1). The L1cI phage did not plate on the LR222 cells carrying the pLC1 plasmid. This suggests that the $6 \mathrm{~kb}$ insert in the pLC1 plasmid possibly carries the wild-type $c$ I gene of L1.

Deletion analysis of $6 \mathrm{~kb}$ PstI fragment To identify the shorter segment of DNA within the previously mentioned $6 \mathrm{~kb}$ PstI fragment carrying the putative wild-type repressor gene of L1, the gel purified $6 \mathrm{~kb}$ Pst I fragment was digested with BamHI. The resulting DNA fragments were ligated with BamHI-digested and Bam $\mathrm{HI} \&$ Pst $\mathrm{I}$ double-digested pSD5S30 DNA. The ligated DNAs were separately transformed into LR222 (L1cIts391) lysogen. The transformants, which formed colonies, both at $32^{\circ}$ and $42^{\circ} \mathrm{C}$, were selected. A couple of the transformants that showed heat-stable properties were obtained from the set where BamHI and PstI double-digested pSD5S30 DNA was used. A restriction analysis of the plasmids that were isolated from 3 such transformants showed that they all carried a $2.1 \mathrm{~kb}$ BamHI-PstI fragment (data not shown). This plasmid was designated pLC2 (Table 1). LR222 (pLC2) also showed immunity to superinfection by $\mathrm{L} 1 c \mathrm{I}^{-}$, which suggests that the $2.1 \mathrm{~kb}$ insert of pLC2 carries the wild-type $c \mathrm{I}$ repressor gene of L1 (Table 1). Further deletion of the $2.1 \mathrm{~kb}$ L1 DNA insert by Sau3AI partial digestion resulted in the loss of immunity to 
Table 1. Plating of $\mathrm{L} 1 \mathrm{cI}^{-}$phages on $M$. smegmatis carrying different plasmids and phages

\begin{tabular}{|c|c|c|c|c|}
\hline \multirow{2}{*}{$\begin{array}{l}\text { M. smegmatis } \\
\text { strains }\end{array}$} & \multirow{2}{*}{$\begin{array}{l}\text { Size of insert } \\
(\mathrm{kb})\end{array}$} & \multirow{2}{*}{$\begin{array}{l}\text { Percent e.o.p } \mathrm{p}^{\mathrm{a}} \\
\quad \text { of } \mathrm{L} 1 \mathrm{cl}^{-}\end{array}$} & \multicolumn{2}{|c|}{ Diameter of Plaques (mm) } \\
\hline & & & $32^{\circ} \mathrm{C}$ & $42^{\circ} \mathrm{C}$ \\
\hline LR222 & - & 100 & 2.12 & 3.10 \\
\hline $\operatorname{LR} 222\left(\mathrm{~L} 1 c \mathrm{I}^{+}\right)$ & - & $<10^{-7}$ & - & - \\
\hline LR222 (pLC1) & 6 & $<10^{-7}$ & - & - \\
\hline LR222 (pLC2) & 2.1 & $<10^{-7}$ & - & - \\
\hline $\mathrm{mc}^{2} 155(\mathrm{pSD} 5 \mathrm{~S} 30)$ & - & 100 & 2.00 & 3.00 \\
\hline $\mathrm{mc}^{2} 155(\mathrm{pCP} 2)$ & 1.4 & 36 & 0.40 & 1.00 \\
\hline
\end{tabular}

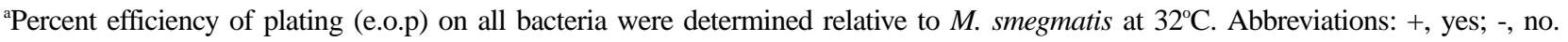
See text for details.

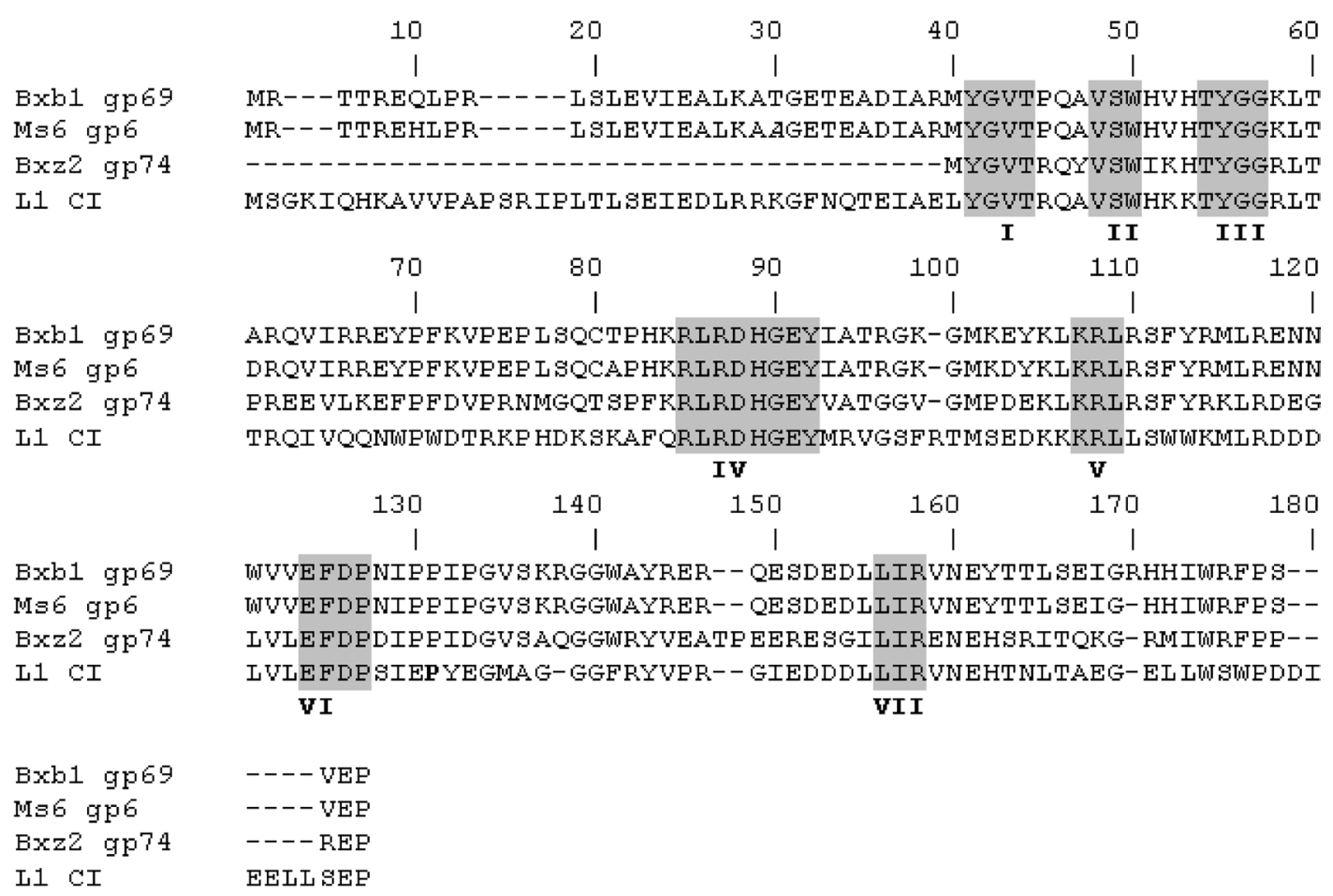

Fig. 1. Alignment of the amino acid sequences of mycobacteriophage-specific repressors. The amino acid sequences of the repressor proteins of Bxb1 (ac. no. AAG59774), L1, MS6 (ac. no. AAG48322), and Bxz2 (ac. no. AAN01828) are aligned by the Clustal W program. Blocks carrying 3-8 identical amino acids are shaded and numbered I to VII. The $131^{\text {st }}$ amino acid proline, which is changed to leucine in the repressor gene of L1cIts391, has been made bold. See text for details.

superinfection by $\mathrm{L}_{1} \mathrm{I}^{-}$(data not shown).

Identification of the repressor gene of $\mathbf{L} 1$ Since the previously mentioned $2.1 \mathrm{~kb}$ L1 DNA fragment carries the repressor gene of $\mathrm{L} 1$, it was sequenced to know more about this negative regulatory gene and other neighboring genes. A sequencing analysis revealed five intact open-reading frames, designated ORF1 to ORF5, in the $2.1 \mathrm{~kb}$ L1 DNA (data not shown). All of the ORFs were transcribed in one direction. To detect which of these ORFs encodes the repressor protein CI of L1, databases were searched against the amino acid sequence of each of the ORFs. Surprisingly, ORF5 was found to be $100 \%$ identical with that of the repressor gene 71 of the homoimmune phage L5 (Hatfull, 2000). Therefore, ORF5 in the $2.1 \mathrm{~kb}$ L1 DNA was designated the $c$ I repressor gene of L1. The ORF1, ORF2, ORF3, and ORF4 were also found to be perfectly identical to gp75, gp74, gp73, and gp72 of L5, respectively. The entire $2.1 \mathrm{~kb}$ L1 DNA sequence is in fact identical to the 43,933-46,039 bp coordinates of the L5 genome (Hatfull, 2000). It was reported earlier that both L1 and L5 have an identical digestion pattern for over 20 restriction enzymes (Lee et al., 1991; our unpublished data), and the former does not yield plaque at $42^{\circ} \mathrm{C}$ (Lee et al., 1991). A variant of $\mathrm{L} 1$ was isolated and grew well at $42^{\circ} \mathrm{C}$ (Chaudhuri et al., 1993). Recently, a 430 bp DNA fragment carrying the early promoters of L1 was also shown to be 
$100 \%$ identical to the DNA region corresponding to the 51,694-52,123 bp coordinates of L5 (Chattopadhyay et al., 2003). All of the data has in fact strengthened the speculation that $\mathrm{L} 1$ is a minor variant of L5 or vice versa.

Other proteins, which show about $40-50 \%$ identities over almost the entire length of L1 repressor are the gp6, gp74, and gp69 of mycobacteriophage MS6, Bxz2, and Bxb1, respectively (Fig. 1). The gp74 is the smallest among these orthologous proteins. The most notable finding is that there are about 31 amino acid residues at the $\mathrm{N}$-terminal end in gp74 that are missing (Fig. 1). Interestingly, the gp6 of Ms6 was more identical (about 96\%) to the repressor of Bxb1 than to either L1 or Bxz2 (Fig. 1). On the other hand, gp74 showed about a 58\% identity to both gp6 and gp69. Apart from these orthologs of the CI protein of L1, a couple of DNA binding proteins also showed a very good degree of similarity within a narrow region (carrying amino acid residues 34-53) of the L1 repressor (discussed below). These results, therefore, suggest that CI of L1, gp6, gp69, and gp74 constitute a new family of repressor proteins of mycobacteriophage origin that possibly evolved from a common ancestor.

Identification of the temperature-sensitive mutation in the repressor gene of L1cIts391 Several ts mutant repressor proteins of phage $\lambda$ were used extensively to elucidate the structure and function of its repressor (Chattopadhyaya and Ghosh, 2003) and to construct a couple of expression vectors (Sambrook et al., 1989). For a similar purpose, a limited library of L1cIts391 genomic DNA was constructed according to the procedure described in Materials and Methods. The plasmids carrying the $1.4 \mathrm{~kb}$ BamHI-SalI fragment were selected for cloning the $t s$ repressor gene of L1cIts391. These plasmids were preferentially selected since the restriction map of the above $2.1 \mathrm{~kb}$ BamHI-PstI L1 DNA fragment showed that the repressor gene of L1 was located within its $1.4 \mathrm{~kb}$ BamHI-SalI fragment (corresponding to $43933-45335 \mathrm{bp}$ co-ordinates of L5). The enzyme $B g I I$ cleaved the latter $1.4 \mathrm{~kb}$ DNA fragment into two smaller fragments, sizes 871 and $532 \mathrm{bp}$, respectively (data not shown). Among the $1.4 \mathrm{~kb}$ insert carrying plasmids, the ones that produced these two fragments upon digestion with $B g l I I$ were, therefore, selected (data not shown). One of them was picked up for further work and designated pBL1. The SalI site of pBL1 was modified by the BamHI linker DNA. The resulting plasmid was designated pBL2. The $1.4 \mathrm{~kb}$ Bam HI fragment of pBL2 was gel-purified and cloned to the identical site of $\mathrm{pSD} 5 \mathrm{~S} 30$. The resulting plasmid, designated $\mathrm{pCP} 2$, was transformed into $M$. smegmatis $\mathrm{mc}^{2} 155$. The efficiency of

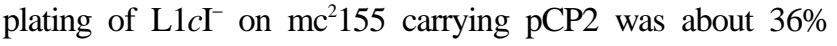
when compared to that on $\mathrm{mc}^{2} 155$ carrying pSD5S30 or nonlysogen at $32^{\circ} \mathrm{C}$ (Table 1). Moreover, the plaque sizes that were produced by $\mathrm{L}_{1 c \mathrm{I}^{-}}$on the lawn of $\mathrm{mc}^{2} 155$ carrying $\mathrm{pCP} 2$ were reduced 5 and 3 folds at $32^{\circ} \mathrm{C}$ and $42^{\circ} \mathrm{C}$, respectively (Table 1). Also, $\mathrm{L} \mathrm{I}^{-}$produced turbid plaques on the lawn of $\mathrm{mc}^{2} 155$ carrying $\mathrm{pCP} 2$ at $32^{\circ} \mathrm{C}$, but not at $42^{\circ} \mathrm{C}$. These results suggest that the $t s$ repressor gene is indeed present in $\mathrm{pCP} 2$, but

\begin{tabular}{|c|c|c|c|c|c|c|c|c|c|c|c|c|c|c|c|c|c|c|c|}
\hline \multirow[b]{2}{*}{ Bxb1 gp69 } & \multicolumn{6}{|c|}{ Helix 1} & \multicolumn{5}{|c|}{ turn } & \multicolumn{8}{|c|}{ Helix 2} \\
\hline & $\mathrm{E}$ & A & D & I A & & & & Y & & & Г & P & Q & A & V & W & $\mathrm{H}$ & & $\mathrm{H}$ \\
\hline Ms6 gp 6 & $E$ & $A$ & D & I A & & & M & Y & & J & T & P & $Q$ & A & V & W & $\mathrm{H}$ & V & $\mathrm{H}$ \\
\hline L1 CI & $Q$ & $\mathrm{~T}$ & E & I $A$ & & & L & Y & & V & $\Gamma$ & $\mathrm{R}$ & $Q$ & A & V & W & $\mathrm{H}$ & K & $K$ \\
\hline 434 Cro & Q & $\mathrm{T}$ & $\mathrm{E}$ & I A & & & K & A & & $\mathrm{V}$ & K & Q & $Q$ & S & I & L & I & E & A \\
\hline P22 Cro & $\hat{Q}$ & $R$ & A & $V A$ & & & & L & & I & S & D & A & A & V & $Q$ & W & K & $\mathrm{E}$ \\
\hline $434 \mathrm{R}$ & $Q$ & A & $\mathrm{E}$ & I A & & & K & V & & $\Gamma$ & & Q & $Q$ & S & I & Q & 工 & $E$ & $\mathrm{~N}$ \\
\hline$\lambda R$ & $Q$ & $\mathrm{E}$ & S & $V A$ & & & & M & & 1 & & Q & S & G & V & A & I & $\mathrm{F}$ & \\
\hline$\lambda$ Cro & $Q$ & $\mathrm{~T}$ & K & $\mathrm{TA}$ & & & & I & & $J$ & $Y$ & $Q$ & S & A & I & K & A & I & \\
\hline P22 R & Q & A & A & L G & & & & V & & J & & $\mathrm{N}$ & V & A & I & 0 & W & $E$ & \\
\hline CAP & $\mathrm{R}$ & $Q$ & E & I G & & & & V & & C & S & $\mathrm{R}$ & $E$ & $\mathrm{~T}$ & V & $R$ & I & & $K$ \\
\hline LexR & $\mathrm{R}$ & & $\mathrm{E}$ & I A & & & & L & & $F$ & & S & P & $\mathrm{N}$ & A & $\mathrm{E}$ & $\mathrm{E}$ & H & I \\
\hline LacR & L & & D & $V A$ & & & & & & V & & Y & Q & $\mathrm{T}$ & V & & V & V & \\
\hline Bxz2 gp 74 & $Q$ & & $\mathrm{E}$ & I A & & & & Y & & V & $\mathrm{T}$ & $\mathrm{R}$ & Q & $\mathrm{Y}$ & V & $\mathrm{w}$ & I & K & \\
\hline
\end{tabular}

Fig. 2. Alignment of helix-turn-helix motifs of proteins. The helix-turn-helix (HTH) motifs of some bacterial and phagespecific regulatory proteins are aligned. Highly conserved residues of HTH motifs are shaded. All of the sequences are available in GenBank. Helix 1, turn, and helix 2 regions of HTH motifs are shown by three rectangles at the top of the figure. The non-assigned amino acid residues of helix 1 of the HTH motif of the putative repressor of Bxz2 are underlined.

its expression is inadequate to completely block the vegetative growth of $\mathrm{L} 1 c \mathrm{I}^{-}$.

A DNA sequence analysis of the $1.4 \mathrm{~kb}$ mutant L1 DNA fragment revealed that the $131^{\text {st }}$ codon, $\mathrm{CCC}$, in the wild-type $c$ I gene of L1 was changed to CTC in the $t s$ repressor gene of L1cIts391 (data not shown). The corresponding amino acid change occurred from proline to leucine in the ts repressor protein (Fig. 1). Interestingly, the deoxynucleotide change in the repressor gene of L1cIts391 created a novel restriction endonuclease site, SacI, which is absent in wild-type L1. The SacI also cleaved the $1.4 \mathrm{~kb}$ BamHI-SalI mutant DNA fragment into two smaller pieces with sizes of 846 and 557 $\mathrm{bp}$, respectively (data not shown).

Identification of the putative domains and motifs in L1 repressor Specific regions of the repressor proteins of $\lambda$ and other phages were shown to direct the specific functions, such as binding with their cognate operators through their HTH motifs, interaction with RNA polymerase, and the interaction between the monomers to form oligomers, etc. (Chattopadhyaya and Ghosh, 2003). To look for these regions in the L1 repressor, if any, its primary sequence was analyzed by several computer programs.

Alignment of gp6, gp69, gp74, and CI protein sequences by Clustal W showed at least 7 blocks (designated I-VII) carrying 3-8 identical amino acid residues (Fig. 1). These identical blocks were possibly assigned for similar work in all 4 proteins. Blocks I and II carried the 'turn' and 'helix 2' (recognition helix) region of the putative helix-turn-helix (HTH) motif, respectively. Alignment of this motif with similar motifs of the proteins of other phages revealed a couple of distinct features, which were not previously identified (Fig. 2). When the putative HTH motifs of the repressors of both $\mathrm{L} 1$ and $\mathrm{Bxb} 1$ were compared, twelve amino 
acids were found common. The helix 2 and turn regions were more homologous than those of the helix 1 (Fig. 2). The HTH motifs of gp69 of Bxb1 and gp6 of Ms6 were 100\% identical, although they are not $100 \%$ similar over the entire length of their amino acid sequences. Interestingly, the gp74 of Bxz2, which produced turbid plaques on $M$. smegmatis (Pedula et al., 2003) and showed a good degree of identity with the repressors of Bxb1 and L1, carried only the helix 2 and turn region of the HTH motif (Fig. 2). A DNA-protein translation program showed that there were 14 extra amino acid residues at the upstream of the N-terminal methionine residue of Bxz 2 gp74 (data not shown). Six of these 14 amino acid residues may potentially form the helix 1 of HTH motif (Fig. 2). It would be interesting to see whether Bxz2 really synthesizes these 14 amino acids prior to the synthesis of gp74 from methionine. However, a further analysis revealed that the helix 1 and turn of the HTH motif of CI of L1 was about $55 \%$ identical to the same phage 434 Cro protein; whereas, helix 2 is about $44 \%$ identical to the same P22 Cro protein (Fig. 2). The most striking feature is that there are three highly charged basic residues in the helix 2 of the HTH motif of CI of L1. Genetic studies showed that substitutions of the acidic or amide derivatives of the acidic amino acids of helix 2 of 434 CI, CAP, lexR to basic or non polar amino acids greatly reduced the binding affinity to their cognate operators (Ebright et al., 1984; Wharton and Ptashne, 1987; Thliveris and Mount, 1992). On the contrary, replacement of the glycine residue of the recognition helix with asparagine increases the affinity of the $\lambda$ repressor to its operator (Hochschild et al., 1986). Furthermore, it was reported that the affinity of the L5 repressor to its operator DNA is low compared to that of the $\lambda$ (Brown et al., 1997) and Bxb1 repressors (Jain and Hatfull, 2000). Taken together, it suggests that three basic amino acids (especially, arginine at position 12 of the helix 2 in the HTH motif) possibly affect the binding of the L1 repressor to the bases at the major groove of its operator.

Block VI and its neighboring sequences strongly control the function of the L1 repressor since the mutations at positions 123,124 , and 131 made it biologically inactive at $42^{\circ} \mathrm{C}$ (Hatfull, 2000; our observation, see above). Similarly, the amino acid residues encompassing block $\mathrm{V}$ are crucial for the repressor proteins function since the L5 repressor, carrying a point mutation at position 108, forms a clear plaque (Hatfull, 2000).

The hydrophobicity plot of CI of L1 revealed that the region carrying block IV and its upstream 13 amino acid residues was much more polar than the flanking regions (data not shown). It was also discovered that the two flanking regions of the L1 repressor, carrying amino acid residues 1-71 and 93-183, are distinctly basic (pI 10.56) and acidic (pI 4.23) in nature, respectively. The Bxb1 repressor also carries both domains at the $\mathrm{N}$ - and C-terminal ends, but they are much weaker than those found in the L1 repressor (data not shown). These basic $\mathrm{N}$-terminal and acidic $\mathrm{C}$-terminal domains are also prevalent in the 434 and $\mathrm{P} 22$ repressor proteins.
The amino acid residues at the downstream of position 131 in the L1 repressor must be vital for its function since the mapping of the different $c$ Its mutations showed that mutations 29 and 578 are located at the right side of mutation 391 (Chaudhuri et al., 1993; data not shown). The function of the block III amino acid residues might also be important, because it was shown that the point mutation in lambda and other phage repressor proteins greatly affect their structure and function (Chattopadhyay and Ghosh, 2003).

Acknowledgments We thank Drs. B. Bloom and Anil Tyagi for the phage and bacterial strains (such as mycobacteriophage L1, M. smegmatis, and plasmid like pSD5S30). The technical assistance of Mr. N. C. Datta is thankfully acknowledged. This research was partly supported by a research grant from C. S. I. R., Govt. of India, New Delhi.

\section{References}

Barletta, R. G., Kim, D. D., Snapper, S. B., Bloom, B. R. and Jacobs, W. R., Jr. (1992) Identification of expression signals of the mycobacteriophage Bxb1, L1 and TM4 using the Escherichia-Mycobacterium shuttle plasmids pYUB75 and pYUB76 designed to create translational fusions to the lacZ gene. J. Gen. Microbiol. 138, 23-30.

Bashyam, M. D., Kaushal, D., Dasgupta, S. K. and Tyagi, A. K. (1996) A study of the mycobacterial transcriptional apparatus: identification of novel features in promoter elements. $J$. Bacteriol. 178, 4847-4853.

Brown, K. L., Sarkis, G. J., Wadsworth, C. and Hatfull, G. F. (1997) Transcriptional silencing by the mycobacteriophage L5 repressor. EMBO J. 16, 5914-5921.

Chaudhuri, B., Sau, S., Datta, H. J. and Mandal, N. C. (1993) Isolation, characterization and mapping of temperaturesensitive mutations in the genes essential for lysogenic and lytic growth of the mycobacteriophage L1. Virology. 194, 166172.

Chattopadhyaya, R. and Ghosh, K. (2003) A comparative threedimensional model of the carboxy-terminal domain of the lambda repressor and its use to build intact repressor tetramer models bound to adjacent operator sites. J. Struct. Biol. 141, 103-114.

Chattopadhyay, C., Sau, S. and Mandal, N. C. (2003) Cloning and characterization of the promoters of temperate mycobacteriophage L1. J. Biochem. Mol. Biol. 36, 586-592.

Dasgupta, S. K., Bashyam, M. D. and Tyagi, A. K. (1993) Cloning and assessment of mycobacterial promoters by using a plasmid shuttle vector. J. Bacteriol. 175, 5186-5192.

Datta, H. J. and Mandal, N. C. (1998) Identification of an early positive regulatory gene of mycobacteriophage L1. J. Gen. Virol. 79, 205-210.

Ebright, R. H., Cossart, P., Gicquel-Sanzey, B. and Beckwith, J. (1984) Mutations that alter the DNA sequence specificity of the catabolite gene activator protein of E. coli. Nature 311, 232-235.

Garcia, M., Pimentel, M. and Moniz-pereira, J. (2002) Expression 
of mycobacteriophage Ms6 lysis genes is driven by two $\sigma 70$ like promoters and is dependent on a transcription termination signal present in the leader RNA. J. Bacteriol. 184, 3034-3043.

Hatfull, G. F. (2000) Molecular Genetics of Mycobacteria. Hatfull, G. F. and Jacob, W. R., Jr. (eds.), pp. 37-54. ASM Press. Washington D.C., USA.

Hochschild, A., Douhan, J. III. and Ptashne, M. (1986) How $\lambda$ Repressor and $\lambda$ Cro distinguish between $\mathrm{O}_{\mathrm{R}} 1$ and $\mathrm{O}_{\mathrm{R}} 3$. Cell 47, 807-816.

Jain, S., Kaushal, D., DasGupta, S. K. and Tyagi, A. K. (1997) Construction of shuttle vectors for genetic manipulation and molecular analysis of Mycobacteria Gene 190, 37-44.

Jain, S. and Hatfull, G. F. (2000) Transcriptional regulation and immunity in mycobacteriophage Bxb1. Mol. Microbiol 38, 971985.

Lee, M. H., Pascopella, L., Jacobs, W. R. Jr. and Hatfull, G. F. (1991) Site-specific integration of mycobacteriophage L5: integration-proficient vectors for Mycobacterium smegmatis, Mycobacterium tuberculosis, and bacille Calmette-Guerin. Proc. Natl. Acad. Sci. USA 88, 3111-3115.

Nesbit, C. E., Levin, M. E., Donnelly-Wu, M. K. and Hatfull, G.
F. (1995) Transcriptional regulation of repressor synthesis in mycobacteriophage L5. Mol. Microbiol. 17, 1045-1056.

Pedulla, M. L., Ford, M. E., Houtz, J. M., Karthikeyan, T., Wadsworth, C., Lewis, J. A., Jacobs-Sera, D., Falbo, J., Gross, J., Pannunzio, N. R., Brucker, W., Kumar, V., Kandasamy, J., Keenan, L., Bardarov, S., Kriakov, J., Lawrence, J. G., Jacobs, W. R. Jr., Hendrix, R. W. and Hatfull, G. F. (2003) Origins of Highly Mosaic Mycobacteriophage Genomes. Cell. 113, 171182.

Ramesh, G. and Gopinathan, K. P. (1995) Cloning and characterization of mycobacteriophage I3 promoters. Indian $\mathrm{J}$. Biochem. Biophys. 32, 361-367.

Sambrook, J., Fritsch, E. F. and Maniatis, T. (1989) Molecular Cloning: A Laboratory Manual. 2nd ed., Cold Spring Harbor Laboratory Press, Cold Spring Harbor, New York, USA.

Thliveris, A. T. and Mount, D. W. (1992) Genetic identification of the DNA binding domain of Escherichia coli LexA protein. Proc. Natl. Acad. Sci. USA 89, 4500-4504.

Wharton, R. P. and Ptashne, M. (1987) A new-specificity mutant of 434 repressor that defines an amino acid-base pair contact. Nature 326, 888-891. 